\title{
43 genes support the lungfish-coelacanth grouping related to the closest living relative of tetrapods with the Bayesian method under the coalescence model
}

\author{
Yunfeng Shan ${ }^{1 *}$, Robin Gras ${ }^{1,2}$
}

\begin{abstract}
Background: Since the discovery of the "living fossil" in 1938, the coelacanth (Latimeria chalumnae) has generally been considered to be the closest living relative of the land vertebrates, and this is still the prevailing opinion in most general biology textbooks. However, the origin of tetrapods has not been resolved for decades. Three principal hypotheses (lungfish-tetrapod, coelacanth-tetrapod, or lungfish-coelacanth sister group) have been proposed.

Findings: We used the Bayesian method under the coalescence model with the latest published program (Bayesian Estimation of Species Trees, or BEST) to perform a phylogenetic analysis for seven relevant taxa and 43 nuclear protein-coding genes with the jackknife method for taxon sub-sampling. The lungfish-coelacanth sister group was consistently reconstructed with the Bayesian method under the coalescence model in 17 out of 21 taxon sets with a Bayesian posterior probability as high as $99 \%$. Lungfish-tetrapod was only inferred from BCLS and BACLS. Neither coelacanth-tetrapod nor lungfish-coelacanth-tetrapod was recovered out of all 21 taxon sets.

Conclusions: Our results provide strong evidence in favor of accepting the hypothesis that lungfishes and coelacanths form a monophyletic sister-group that is the closest living relative of tetrapods. This clade was supported by high Bayesian posterior probabilities of the branch (a lungfish-coelacanth clade) and high taxon jackknife supports.
\end{abstract}

\section{Background}

The origin of land vertebrates (tetrapods) has not been fully resolved. Since the discovery of the "living fossil" in 1938, Latimeria chalumnae [1,2], the last discovered surviving species of a lineage of lobe-finned fish, has generally been considered the closest living relative of the land vertebrates, the missing link between aquatic and terrestrial vertebrates. This is still the prevailing opinion in most general biology textbooks [3]. The origin of tetrapods always has considerable popular interest in public and academic fields since the legendary fish discovery. Three hypotheses have been proposed for the phylogenetic relationship: e.g., lungfish-tetrapod

\footnotetext{
* Correspondence: shan@cs.dal.ca

'School of Computer Science, University of Windsor, 401 Sunset Avenue, Windsor, ON N9B 3P4, Canada

Full list of author information is available at the end of the article
}

(Hypothesis 1, Figure 1a), coelacanth-tetrapod (Hypothesis 2, Figure 1b), or, lungfish-coelacanth sister group (Hypothesis 3, Figure 1c). The coelacanth-lungfish-tetrapod trichotomy (Figure 1d) is not generally considered a hypothesis.

Based on comparative morphological and paleontological studies, the lungfish were historically thought to be the closest living relatives of tetrapods $[4,5]$, but the coelacanths were purported to have that claim $[1,6,7]$ since its discovery in 1938, whereas the coelacanths and lungfish sister group (Tree III) was also proposed [8-10].

Over the last two decades, single genes and whole mitochondrial genomes have been sequenced with a view to inferring phylogenetic relationships. Lungfish as the closest relatives of tetrapods were supported by single genes [11-15] and mitochondrial whole genomes [16-19], the coelacanth as the closest living sister group

\section{C) Biomed Central}




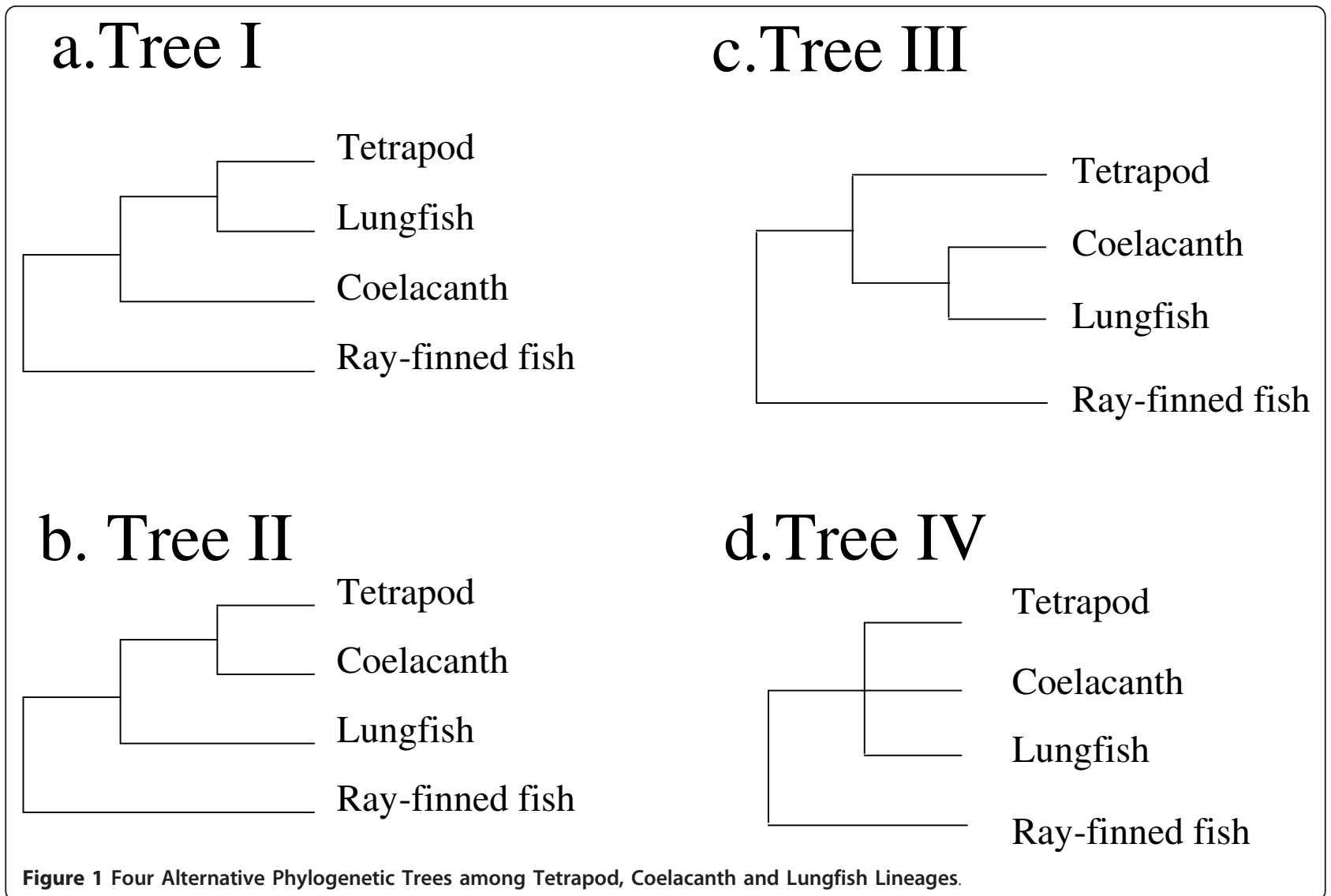

of tetrapods was preferred by single genes [20], and coelacanth-lungfish sister group relationship was suggested by the single gene [13] and the mitochondrial whole genome $[17,19]$, while an unresolved coelacanth-lungfish-tetrapod trichotomy was shown by the $12 \mathrm{~S}$ rRNA gene [12].

Recently, this question was reinvestigated. The result using 44 genes with the concatenation genome-scale approach [21] was an unresolved trichotomy. Another from two recombination activating genes (Rag1 and Rag2) supported lungfish and not the coelacanth as the closest living relative of the tetrapods [15]. Our previous study provided strong evidence in favor of rejecting Hypothesis 2, but weak evidence to support Hypothesis 3 based on 43 genes with three common phylogenetic methods and three genome-scale approaches [22,23].

Although many morphological, paleontological and molecular phylogenetic studies have attempted to resolve this question, the results have so far not discovered unequivocal evidence as to whether either the coelacanth or the lungfish is the closest living relatives of tetrapods or that both lineages are equally closely related to tetrapods. Therefore, the origin of tetrapods continues to be debated and still is one of the longest standing major questions in vertebrate evolution.
BEST implements a Bayesian hierarchical model to jointly estimate gene trees and the species tree from multilocus sequences [24]. The procedure applies the same substitution models as those used in traditional phylogenetics and coalescent theory to explain genealogical signals from species trees to gene trees and from gene trees to sequence data, forming a complete stochastic model to estimate gene trees, species trees and species divergence times simultaneously [25]. The model is based on the assumption that gene trees are correlated due to being come from a single species tree and therefore should be estimated jointly [25]. It provides a new approach for estimating species phylogenies within the popular Bayesian phylogenetic program MrBayes [26]. BEST was applied to a prominent yeast phylogenomics data set and have shown that it is more efficient in estimating the species tree than concatenation is in estimating the gene tree [27]. With simulation data, analysis of the same DNA sequences by concatenation using either Bayesian or maximum-likelihood methods reconstructed the wrong tree with high confidence, whereas the BEST analysis converged on the correct tree with high confidence [27].

To provide more evidence to resolve the origin of tetrapods, we used here the Bayesian method under the 
coalescence model with a newly published program (Bayesian Estimation of Species Trees, or BEST) for genome-scale phylogenetic analysis [24-27] and the jackknife method for taxon sub-sampling to analyze all 43 nuclear protein-encoding genes that are currently available in Genbank, having considered the results of our previous study [23] using three other genome-scale approaches with all three commonly used phylogenetic methods together. Seven taxa include Mammal (M), Bird (B), Amphibian (A), Coelacanth (C), Lungfish (L), Ray-finned Fish (R), and Shark (S).

\section{Materials and methods}

\section{Sequence Collection}

The sequences of 43 nuclear protein-encoding genes were downloaded from GenBank through the National Center for Biotechnology Information http://www.ncbi.nlm.nih. gov/ using the program SeqMiner.pl [28]. These 43 genes were previously analyzed using the genome-scale approach of concatenated genes; however, the sequence length of some genes was different [Additional file 1: Supplemental Table S1]. One gene (FSCN1) is omitted because some taxa lack its sequences in GenBank. In order to compare the results with the genome-scale concatenated multiple gene approach [21], the same seven taxa were included: Mammal, Bird, Amphibian, Coelacanth, Lungfish, Rayfinned Fish, and Shark. The species examined included human (Homo sapiens), bird (Gallus gallus), amphibian (Xenopus laevis), coelacanth (mostly Latimeria chalumnae, with a few L. menadoensis), lungfish (mostly Protopterus dolloi and P. aethiopicus) with a few Neoceratodus forsteri and Lepidosiren paradoxa), ray-finned fishes (Danio rerio), and cartilaginous fishes (represented by Scyliorhinus canicula) [21].

\section{Phylogenetic Analysis}

Sequences of an individual gene were aligned using ClustalX with default settings [29]. All alignments of single genes were manually edited to exclude insertions or deletions and uncertain positions for further analysis. The BEST phylogenetic analysis software (version 1.1) with the Bayesian method under the coalescence [24] was used for tree inference under the GTR $+\Gamma+I$ model and four simultaneous Markov chains for 20 million generations, starting with random initial trees and sampling every 2000 generations. The burnin value was set to 100 . The majority rule consensus tree was generated using the remaining trees with posterior probability plotted on each node.

\section{Taxon Jackknife Sub-sampling}

We used a jackknife approach to sub-sample six, five and four taxa from seven taxa with permutation and combination. The debate over taxon sampling has not terminated. On the one hand, the accuracy was enhanced dramatically with the addition of taxa [30]. On the other hand, adding taxa can reduce accuracy and increase the probability of distorting the tree topology [30]. Adding characters can always increase the accuracy [30-32]. So, as many genes as possible should be included. The sequence data of 43 genes that are all currently available in GenBank were used in this study. Sequence data sets are available upon request.

\section{Chi-square Test}

The statistically significant difference in the Bayesian posterior probabilities for the branch of the lungfishcoelacanth or the taxon jackknife support averages between the six-, five- and four-taxon sets was analyzed by means of the chi-square test.

\section{Results}

Tree III was inferred with 90\% Bayesian posterior probability of the branch of lungfish-coelacanth for seven taxon set (Figure 2 and Table 1). Four of five six-taxon sets recovered tree III with Bayesian posterior probabilities for the branch of lungfish-coelacanth ranging from 77 to $93 \%$. The exception was MBCLRS, which recovered an alternative tree (Table 1). Seven of the nine five-taxon sets inferred tree III, but BACLS recovered tree I and ACLRS reconstructed an alternative tree. Although BCLS recovered tree I, all the other five fourtaxon sets inferred tree III (Table 1).

Taxon jackknife support was $81.0 \%$ for tree III, 9.5\% for tree I or an alternative tree other than tree I - IV, and zero for tree II and tree IV with the Bayesian method under the coalescence model for all 21 taxon sets (Table 1).

Bayesian posterior probabilities for the branch of lungfish-coelacanth were 85.0, 64.0, and 70.4\% and taxon

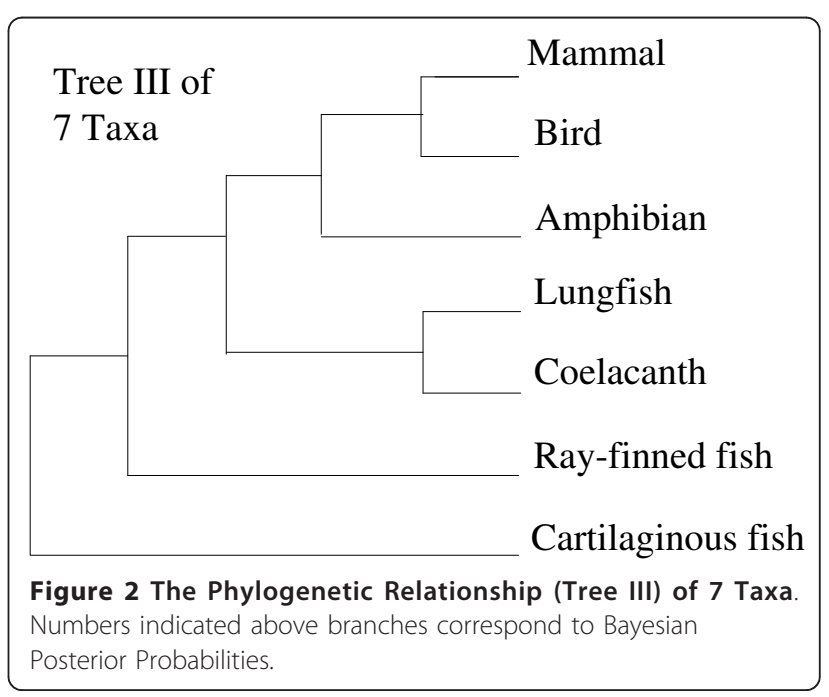


Table 1 Tree Types, Bayesian Posterior Probability of the Branch of Lungfish-Coelacanth (tree III) or LungfishTetrapods (Tree I) for 7, 6, 5 and 4-Taxon Sets, and Taxon Jackknife Supports with the Bayesian Method under the Coalescence Model

\begin{tabular}{|c|c|c|}
\hline Taxon Set & Tree Type & Probability \\
\hline \multicolumn{3}{|l|}{7 taxon set } \\
\hline MBACLRS & III & $90 \%$ \\
\hline \multicolumn{3}{|l|}{6 taxon sets } \\
\hline BACLRS & III & $90 \%$ \\
\hline MACLRS & III & $80 \%$ \\
\hline MBACLR & III & $93 \%$ \\
\hline MBACLS & III & $77 \%$ \\
\hline MBCLRS & AT & $\mathrm{n} / \mathrm{a}$ \\
\hline \multicolumn{3}{|l|}{5 taxon sets } \\
\hline ACLRS & AT & $\mathrm{n} / \mathrm{a}$ \\
\hline BACLR & III & $49 \%$ \\
\hline BACLS & 1 & $43 \%$ \\
\hline BCLRS & III & $49 \%$ \\
\hline MACLR & III & $97 \%$ \\
\hline MACLS & III & $64 \%$ \\
\hline MBCLR & III & $45 \%$ \\
\hline MBCLS & III & $82 \%$ \\
\hline MCLRS & III & $62 \%$ \\
\hline \multicolumn{3}{|l|}{4 taxon sets } \\
\hline ACLR & III & $99 \%$ \\
\hline ACLS & III & $67 \%$ \\
\hline BCLR & III & $40 \%$ \\
\hline BCLS & । & $89 \%$ \\
\hline MCLR & III & $73 \%$ \\
\hline MCLS & III & $73 \%$ \\
\hline \multirow[t]{5}{*}{ JKF: } & III (17/21) & $81.00 \%$ \\
\hline & I (2/21) & $9.50 \%$ \\
\hline & AT $(2 / 21)$ & $9.50 \%$ \\
\hline & $\|$ & 0 \\
\hline & IV & 0 \\
\hline
\end{tabular}

Notes:

The taxa included: Mammal (M), Bird (B), Amphibian (A), Coelacanth (C), Lungfish (L), Ray-finned Fish (R), and Shark (S); JKF = Taxon jackknife supports (\%); $\mathrm{AT}=$ alternative tree; $\mathrm{n} / \mathrm{a}=$ not available.

jackknife support averages were $83.3,77.8$, and $80.0 \%$ for the six-, five-, and four-taxon sets, respectively (Table 1). The chi-square test showed no significant differences among the taxon sampling sets. The results showed that taxon sampling had no significant effect on phylogenetic inference for the taxon sets.

\section{Discussion}

Tree III is consistently reconstructed with the Bayesian method under the coalescence model in 17 out of 21 taxon sets with a Bayesian posterior probability as high as $99 \%$. Tree I was inferred only from BCLS and BACLS, and two alternative trees were recovered from ACLRS and MBCLRS (Table 1). Therefore, we provide strong evidence to support Hypothesis 3, namely that coelacanths and lungfish form a monophyletic group that is the phylogenetically closest living relatives of tetrapods (Tree III). Our results agree with those of other studies in terms of the morphological, palaeontological and molecular analyses below. The coelacanth and lungfish sister group relationship was supported by the single gene [13] and the whole mitochondrial genome [19], and by the nuclear $28 \mathrm{~S}$ ribosomal RNA gene [17]. This relationship was also proposed in comparative morphological and paleontological studies [8-10].

Recently, an investigation using 44 genes with a concatenation genome-scale approach showed an unresolved trichotomy [21]. Another result from two genes supported lungfish and not the coelacanth as the closest living relative of the tetrapods [15]. In our previous study [22,23], tree II received significantly lower support than tree I or tree III and, evidently, lower taxon jackknife probabilities with all the phylogenetic methods and genome-scale approaches. The supports for tree III were significantly higher than those for tree I for only two out of 63 events, and taxon jackknife probabilities for tree III were slightly higher than those for tree I with MP, but the differences in supports and taxon jackknife probabilities between tree III and tree I are not as obvious as those between tree II and tree III/I. Therefore, the results in our previous study provide strong evidence to reject Hypothesis 2 that coelacanth is the closest living relative of tetrapods, but only weak support for Hypothesis 3 based on phylogenetic analysis of 43 genes with those three common methods and those three genome-scale approaches yet at that time $[22,23]$. Our results in this study also provide further strong evidence in favor of rejecting Hypothesis 2 because none of 21 taxon sets recovers tree II. Recently major palaeontological studies proposed that lungfishes are the closest living relatives of the tetrapods or alternatively, that coelacanths and lungfishes form a monophyletic sister group that is equally closely related to the tetrapods $[33,34]$. The cause of this puzzle is the fact that the divergence of coelacanth and lungfish happened over a relatively short period within a small (20-30 millions years) window in time around 400 million years ago $[3,5]$. This results in little time and opportunity for lineage-specific molecular changes to happen, yet considerable time and opportunity for multiple and parallel changes and their accumulation since the origin of these two lineages [3]. For this challenging phylogenetic question, therefore, it was very difficult to achieve high resolution using ad hoc molecular phylogenetic methods 
and algorithms given that the available sequence data set of genes were currently very limited before the publication of the BEST program using the Bayesian method under the coalescence model [24]. However, we would like to point out that the species tree inferred from gene trees using the BEST program achieves high resolution, but is not always correct for all cases. The wrong species trees, such as those of ACLRS and MBCLRS, may be recovered from gene trees (Table 1). Therefore, the jackknife method for taxon sub-sampling is recommended to obtain further statistical confidence with jackknife support values. Additionally, this approach is newly published, it is not surprising that it has not been used widely in its early stage compared with the popular concatenation approach. Some caution should be kept. However, its use is strongly encouraged based on our study and other [27].

\section{Conclusions}

This study provides strong evidence in favor of accepting Hypothesis 3, namely that the lungfish and coelacanth form a monophyletic sister group and that the sister group should be the phylogenetically closest living relatives of tetrapods. These conclusions are supported by high Bayesian posterior probabilities for the branch (a lungfish-coelacanth clade) and high taxon jackknife supports based on the genome-scale phylogenetic analysis of 43 genes using the latest program (BEST) $[24,25,27]$ with the Bayesian method under the coalescence model and the jackknife method for taxon subsampling.

\section{Additional material}

Additional file 1: Supplementary Table S1. List of 43 gene names and their lengths (Number of amino acid positions encoded by the gene).

\section{Acknowledgements}

We thank Richard Winterbottom at the Department of Natural History, Royal Ontario Museum, 100 Queen's Park, Toronto, Ontario M5 S 2C6, Canada for valuable suggestions, as well as we thank Adam Aspinall in our lab for reading this paper and providing valuable comments. This work was partially supported by the NSERC grant ORGPIN 341854, the CRC grant 950-2-3617 and the CFI grant 203617. We thank two anonymous reviewers for their good comments for improving the manuscript.

\section{Author details}

${ }^{1}$ School of Computer Science, University of Windsor, 401 Sunset Avenue, Windsor, ON N9B 3P4, Canada. ${ }^{2}$ Department of Biological Sciences, University of Windsor, 401 Sunset Avenue, Windsor, ON N9B 3P4, Canada.

\section{Authors' contributions}

YS and RG conceived and designed the experiments. YS analyzed the data. YS and RG wrote the paper. Both authors read and approved the final manuscript.

\section{Competing interests}

The authors declare that they have no competing interests.
Received: 25 September 2010 Accepted: 7 March 2011

Published: 7 March 2011

\section{References}

1. Fritzsch B: Inner ear of the coelacanth fish Latimeria has tetrapod affinities. Nature 1987, 327:153-154.

2. Thomson KS: Living fossil New York: W. W. Norton \& Company; 1991

3. Meyer A: Molecular evidence on the origin of tetrapods and the relationships of the coelacanth. Trends Ecol Evol 1991, 10:111-116.

4. Panchen AL, Smithson TS: Character diagnosis, fossils and the origin of tetrapods. Biol Rev 1991, 62:341-438.

5. Ahlberg PE: Postcranial stem tetrapod remains from the Devonian Scat Craig, Morayshire. Scotland Zool J Linn Soc 1991, 103:241-287.

6. Long JA: A new rhizodontiform fish from the Early Carboniferous of Victoria, Australia, with remarks on the phylogenetic position of the group. J Vert Paleontol 1989, 9:1-17.

7. Zhu M, Schultze HP: The oldest sarcopterygian fish. Lethaia 1997, 30:293-304.

8. Northcutt RG: Lungfish neural characters and their bearing on sarcoptergian phylogeny. In The biology and evolution of lungfishes. Edited by: Bemis WE, Burggren WW, Kemp NE. New York: Alan R Liss; 1986:277-297.

9. Chang MM: "Rhip idistians," dipnoans and tetrapods. In Origins of the higher groups of tetrapods: controversy consensus. Edited by: Schultze HP, Trueb L. New York: Cornell University Press; 1991:3-28.

10. Forey $\mathrm{PL}$, Gardiner $\mathrm{BG}$, Patterson C: The lungfish, the coelacanth and the cow revisited. In Origins of the higher groups of tetrapods: controversy consensus. Edited by: Schultze HP, Trueb L. New York: Cornell University Press; 1991:145-172.

11. Meyer A, Wilson AC: Origin of tetrapods inferred from their mitochondrial DNA affiliation to lungfish. J Mol Evol 1990, 31:359-364.

12. Meyer A, Dolven SI: Molecules, fossils, and the origin of tetrapods. J Mol Evol 1992, 35:102-113.

13. Yokobori Al, Hasegawa M, Ueda T, Okada N, Nishikawa K, Watanabe K: Relationship among coelacanths, lungfishes, and tetrapods: a phylogenetic analysis based on mitochondrial cytochrome oxidase I gene sequences. J Mol Evol 1994, 38:602-609.

14. Venkatesh B, Erdmann MV, Brenner S: Molecular synapomorphies resolve evolutionary relationships of extant jawed vertebrates. Proc Natl Acad Sci USA 2001, 98:11382-11387.

15. Brinkmann $H$, Venkatesh B, Brenner S, Meyer A: Nuclear protein-coding genes support lungfish and not the coelacanth as the closest living relatives of land vertebrates. Proc Natl Acad Sci USA 2004, 101:4900-4905

16. Hedges SB, Hass CA, Maxson LR: Relations of fish and tetrapods. Nature 1993, 363:501-502

17. Zardoya R, Meyer A: Evolutionary relationships of the coelacanth, lungfish, and tetrapods based on the $28 \mathrm{~S}$ ribosomal RNA gene. Proc Natl Acad Sci USA 1996, 93:5449-5454

18. Zardoya R, Meyer A: The complete DNA sequence of the mitochondrial genome of a 'living fossil,' the coelacanth (Latimeria chalumnae). Genetics 1997, 146:995-1010.

19. Zardoya R, Cao Y, Hasegawa M, Meyer A: Searching for the closest living relative(s) of tetrapods through evolutionary analyses of mitochondrial and nuclear data. Mol Biol Evol 1998, 15:506-517.

20. Gorr T, Kleinschmidt T, Fricke $\mathrm{H}$ : Close tetrapod relationships of the coelacanth Latimeria indicated by haemoglobin sequences. Nature 1991, 351:394-397.

21. Takezaki N, Figueroa F, Zaleska-Rutczynska Z, Takahata N, Klein J: The Phylogenetic Relationship of Tetrapod, Coelacanth, and Lungfish Revealed by the Sequences of Forty-Four Nuclear Genes. Mol Biol Evol 2004, 21:1512-1524.

22. Shan Y, Li X: Maximum Gene-support Tree. Evolotionary Bioinformatics 2008, 4:181-191.

23. Shan Y, X Li XQ, Gras R: Three Genome-scale Approaches Rejected the Hypothesis that Coelacanth Is the Closest Living Relative of Tetrapods. arXiv:0910.1949v1, [q-bio.PE].

24. LiU L: BEST: Bayesian estimation of species trees under the coalescent model. Bioinformatics 2008, 24:2542-2543.

25. Liu L, Pearl DK: Species trees from gene trees: reconstructing Bayesian posterior distributions of a species phylogeny using estimated gene tree distributions. Syst Biol 2007, 56:504-514. 
26. Ronquist F, Huelsenbeck JP: MrBayes 3: Bayesian phylogenetic inference under mixed models. Bioinformatics 2003, 19:1572-1574.

27. Edwards SV, Liu L, Pearl DK: High-resolution species trees without concatenation. Proc Natl Acad Sci USA 2007, 104:5936-5941.

28. Shan Y, Gras R: Genome-wide EST data mining approaches to resolving incongruence of molecular phylogenies. Advances in Computational Biology Arabnia HA: New York: Springer; 2010, 237-243.

29. Thompson JD, Gibson TJ, Plewniak F, Jeanmougin F, Higgins DG: The ClustalX windows interface: flexible strategies for multiple sequence alignment aided by quality analysis tools. Nucleic Acids Res 1997, 24:4876-4882.

30. Poe S, Swofford DL: Taxon sampling revisited. Nature 1999, 398:299-300.

31. Graybeal A: Is it better to add taxa or characters to a difficult phylogenetic problem? Syst Biol 1998, 47:9-17.

32. Rosenberg MS, Kumar S: Incomplete taxon sampling is not a problem for phylogenetic inference. Proc Natl Acad Sci USA 2001, 98:10751-10756.

33. Zhu M, Yu XB, Ahlberg PE: A primitive sarcopterygian fish with an eyestalk. Nature 2001, 410:81-84.

34. Zhu M, Yu X: A primitive fish close to the common ancestor of tetrapods and lungfish. Nature 2002, 418:767-770.

doi:10.1186/1756-0500-4-49

Cite this article as: Shan and Gras: 43 genes support the lungfishcoelacanth grouping related to the closest living relative of tetrapods with the Bayesian method under the coalescence model. BMC Research Notes 2011 4:49.

\section{Submit your next manuscript to BioMed Central} and take full advantage of:

- Convenient online submission

- Thorough peer review

- No space constraints or color figure charges

- Immediate publication on acceptance

- Inclusion in PubMed, CAS, Scopus and Google Scholar

- Research which is freely available for redistribution

Submit your manuscript at www.biomedcentral.com/submit 